\title{
Actuator Fault-Tolerant Control Applied to Three-Tank System
}

\author{
Mondher Amor (D), Taoufik Ladhari, Salim Hadj Said, and Faouzi M’Sahli \\ Industrial Systems Study and Renewable Energy Unit, National Engineering School of Monastir, University of Monastir, \\ Ibn El Jazzar Street, Skanes 5019, Monastir, Tunisia \\ Correspondence should be addressed to Mondher Amor; mondheramor@yahoo.fr
}

Received 23 March 2020; Accepted 27 April 2020; Published 18 May 2020

Guest Editor: Rongwei Guo

Copyright (c) 2020 Mondher Amor et al. This is an open access article distributed under the Creative Commons Attribution License, which permits unrestricted use, distribution, and reproduction in any medium, provided the original work is properly cited.

\begin{abstract}
This paper focuses on the application of an active fault-tolerant control (AFTC) to a real nonlinear system using analytic redundancy in case of actuator faults. This approach is composed of the fault detection, isolation, and estimation (FDIE) module and a control compensation module. A high-gain observer (HGO) is used for FDIE; a new control law is then reconstructed by the addition of the estimated actuator fault magnitude to the nominal control law. Experimental results highlight the performance of the proposed approach when it is applied to the hydrographic plant.
\end{abstract}

\section{Introduction}

The modern technical process relies on an advanced control system to meet the increased requirement of safety and reliability. The probability of fault occurrence increases with the complexity of the industrial process. For safety-critical systems, such as aircraft, nuclear, chemical, and power production plants, the control system must include automatic supervision of process control to detect and isolate faults as early as possible and to tolerate some component malfunctions [1-4]. In such systems, since the consequence of a minor fault can be catastrophic, the demand for reliability, safety, and fault tolerance is generally high. These types of control systems are often known as the fault-tolerant control (FTC). FTC can be performed by passive methods or by active methods. Passive methods use a robust control technique to ensure that a closed-loop system remains insensitive to certain faults. However, in active methods, a new control system is redesigned by using the desired performance in the faulty-free system.

An AFTC has two subsystems: a module for fault detection, isolation, and estimation (FDIE) and another one for the reconfigurable control (RC). In this paper, the proposed approach is based on the online fault detection, isolation, and estimation of actuator faults using a high-gain observer. Then, a computation of a new control law different from the nominal one is performed in order to compensate for this fault effect and to maintain the three-tank system outputs to their nominal values.

The three-tank plant is a good prototype of many applications in industrial processes, such as water treatment, food industry, chemical and petrochemical plants, and oil and gas systems [1-4]. It is used in water conditioning systems, which provide the user with an abundant supply of luxuriously conditioned water, and in the craft brewing system. This benchmark system represents a rich ground to serve as a test environment for linear and nonlinear control, fault detection and isolation (FDI), and fault-tolerant control (FTC).

The fault detection and isolation is an important research area in system control due to the improvements that it can be reached in terms of safety and reliability of the process. To detect and isolate actuator and component faults around the operating point, authors have proposed in [5] a decoupled linear observer. Many model-based approaches are applied to the hydrographic system for fault detection and estimation [6, 7]. More recently, a bank of observers to detect and isolate actuator and sensor faults around multiple operating points is suggested in [8]. In our previous work [9], we have proposed a high-gain observer to estimate the 
actuator fault magnitude, and eventual hardware implementation is achieved.

In the FTC field, despite several methods have been already applied to the three-tank system, few fault accommodation techniques have been considered. Among the model-based approaches that have been applied to the threetank system, we find fuzzy approaches for fault accommodation [10] and flatness-based active fault-tolerant control $[11,12]$. In [13], authors have dealt with FTC for actuator faults; they have proposed an online estimation of an eventual actuator fault and the addition of a new control law for the three-tank system. Other researchers have focused on fault diagnosis and accommodation for sensor and actuator faults, and they have proposed an analytical redundancy method to solve the drawbacks of the hardware redundancy such as cost and space [14]. All these previous works have developed FTC schemes for the linearized threetank model; this means that their approaches are valid only around the operating point. To overcome previous misachievements, this paper focuses on the application of the actuator tolerant-fault control using a nonlinear MIMO model of the three-tank system. The proposed approach is based on the online actuator fault detection using a highgain observer. The estimates are used in a new control law, which can fastly react to the failure by adding the recovered fault magnitude. The main contribution of this paper compared to previous works is to potentially mitigate the fault-detection delay as well as the fault-compensation time after the fault occurrence.

The remainder of the paper is organized as follows: in Section 2, we represent the three-tank process which is used to illustrate the performance of the proposed AFTC and its dynamical model. The fault detection and estimation module (FDE) and the proposed observer are detailed in Section 3. Section 4 is devoted to the proposed AFTC. Finally, the experimental results and discussion are reported in Section 5 before an illustration of the conclusion remarks.

\section{Three-Tank System Presentation}

2.1. Plant Description. The hydrographical process described in [15] consists of 3 columns $T_{1}, T_{2}$, and $T_{3}$, with the same section $S$, coupled serially by transfer valves and which can be drained into a reservoir by leakage valves. These leakage valves denoted $V_{l 1}, V_{l 2}, V_{l 3}$, and $V_{e}$ have identical effective sections $S_{n}$. Two rotary valves $V_{t 12}$ and $V_{t 23}$ can be used to change the channel section and therefore to change the flow characteristics between the columns. Each leakage valve can be used as a manually adjustable disturbance.

Tanks 1 and 3 are supplied with fluid via 2 pumps of maximum flow $Q_{\max }$. The maximum flow rate of the pump $\left(6.66 \times 10^{-5} \mathrm{~m}^{3} / \mathrm{s}\right)$ is reached when a voltage of $12 \mathrm{~V}$ is applied to the pump. Each column is equipped with a pressure sensor giving the liquid level in the tank. The sensors are calibrated to provide output signals ranging from 0 to $5 \mathrm{~V}$ corresponding to a change in water level from 0 to $100 \mathrm{~cm}$ for each column. The experimental system shown in Figure 1 is equipped with sensors and actuators that communicate via an acquisition card and a computer.

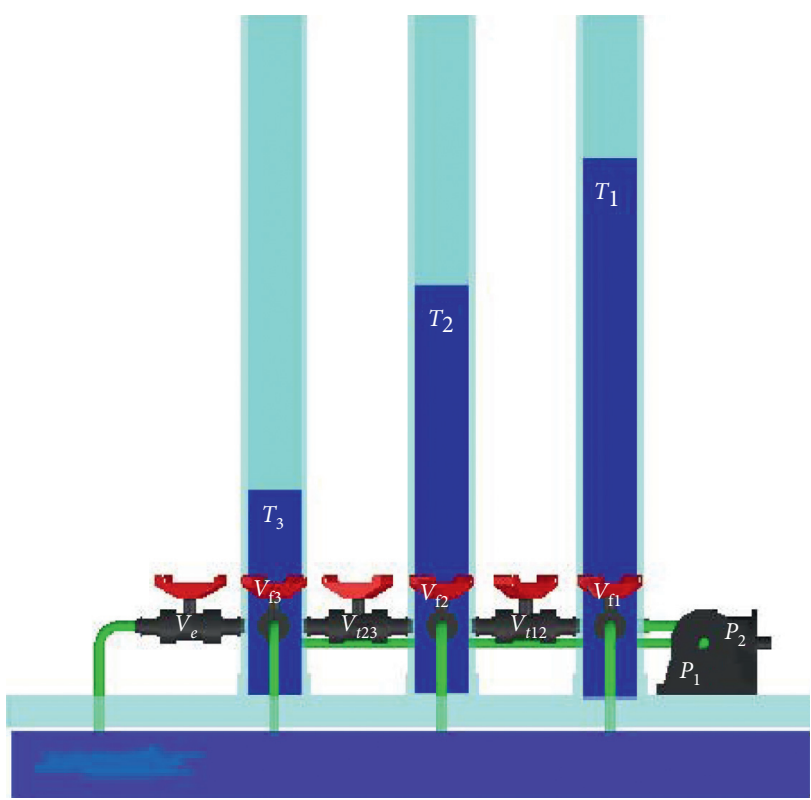

FIgUre 1: Full structure of the computer design plant.

2.2. Mathematical Model. The state equations are obtained by writing that the variation of the volume of water in tank $i(i=1$ : 3 ) is equal to the sum of the inflows minus the sum of the outflows. Using the mass equations (flow equilibrium), the system can be easily represented by the following equation:

$$
\dot{V}_{i}=S_{i} \cdot \frac{\mathrm{d} h_{i}}{\mathrm{~d} t}=\sum Q_{\mathrm{in}, i}-\sum Q_{\mathrm{out}, i}
$$

where $\sum Q_{\text {in }, i}$ and $\sum Q_{\text {out }, i}$ represent the total liquid inflows and outflows in tank $i$, respectively. Then, the mathematical model is specified by the following mass balance equations:

$$
\left\{\begin{array}{l}
\dot{h}_{1}(t)=\frac{1}{S}\left(Q_{1}(t)-Q_{12}(t)-Q_{l 1}(t)\right) \\
\dot{h}_{2}(t)=\frac{1}{S}\left(Q_{12}(t)-Q_{23}(t)-Q_{l 2}(t)\right) \\
\dot{h}_{3}(t)=\frac{1}{S}\left(Q_{2}(t)+Q_{23}(t)-Q_{e}(t)-Q_{l 3}(t)\right)
\end{array}\right.
$$

where $t$ represents the time, $h_{1}, h_{2}$, and $h_{3}$ represent the liquid levels in each tank, $S$ represents the cross section of the tanks, $Q_{1}$ and $Q_{2}$ designate, respectively, the flow rates of pumps $P_{1}$ and $P_{2}, Q_{i j}$ denote the flow rates between tank $T_{i}$ and $T_{j}$, and $Q_{l i}$ represents the leakage flow of the corresponding tank when its leak valve is open.

The flows $Q_{i j}$ and $Q_{e}$ in (2) are given by Torricelli's law as follows:

$$
\begin{aligned}
& Q_{i j}(t)=a_{z i} S_{n} \operatorname{sgn}\left(h_{i}-h_{j}\right) \sqrt{2 g\left|h_{i}-h_{j}\right|}, \\
& Q_{e}(t)=a_{z 3} S_{n} \sqrt{2 g h_{3}},
\end{aligned}
$$

where $a_{z i}$ is the outflow coefficient, $\operatorname{sgn}(\cdot)$ is the sign of the argument, and $g$ is the acceleration of gravity. Consequently, the nonlinear $3 \mathrm{~T}$ model is given as follows: 


$$
\left\{\begin{array}{l}
\frac{\mathrm{d} h_{1}}{\mathrm{~d} t}=-c_{1} \operatorname{sign}\left(h_{1}-h_{2}\right) \sqrt{\left|h_{1}-h_{2}\right|}+\frac{Q_{1}}{S}, \\
\frac{\mathrm{d} h_{2}}{\mathrm{~d} t}=c_{1} \operatorname{sign}\left(h_{1}-h_{2}\right) \sqrt{\left|h_{1}-h_{2}\right|}-c_{2} \operatorname{sign}\left(h_{2}-h_{3}\right) \sqrt{\left|h_{2}-h_{3}\right|}, \\
\frac{d h_{3}}{d t}=c_{2} \operatorname{sign}\left(h_{2}-h_{3}\right) \sqrt{\left|h_{2}-h_{3}\right|}-c_{3} \sqrt{h_{3}}+\frac{Q_{2}}{S},
\end{array}\right.
$$

where $c_{i}$ represents the system parameter given by

$$
c_{i}=\frac{1}{S} a_{z i} S_{n} \sqrt{2 g} ; \quad i=1, \ldots, 3 .
$$

2.3. Three-Tank System Fault Representation. The three-tank laboratory system is considered as a rich ground to serve as a test environment for the FTC. It is used as a benchmark system that can be affected by various additive and/or multiplicative actuator faults.

An actuator fault can be represented by

$$
u_{i}^{f}(k)=\alpha_{i} u_{i}(k)+u_{i 0}, \quad i=1,2,
$$

where $u_{i}^{f}$ and $u_{i}$ represent the faulty and the normal control action of the $i$ th pump, respectively.

The constant offset is denoted by $u_{i 0}$, and $0 \leq \alpha_{i} \leq 1$ denotes a gain degradation of the $i$ th actuator.

In this paper, we have focused only on a partial reduction in actuator efficiency. However, this method fails in the case of complete loss of an actuator, and hardware redundancy is needed.

\section{Fault Detection and Estimation (FDE)}

3.1. Problem Statement. It is important to be able to carry out the fault detection and estimation before the faults induce a catastrophic effect on the system performance. The observerbased approach is used to generate both residual signals corresponding to the difference between measured and estimated signals and the estimated actuator faults. However, the resultant residual will be equal to zero in case of the unfaulty system. The residual signal is compared to a fixed threshold well chosen to avoid false alarms. To compensate for actuator faults, authors have used in the previous work [9] a high-gain observer whose role is to estimate the actuator fault magnitude which is added to the nominal control law as it is shown in Figure 2.

\subsection{Observer Design}

3.2.1. Basic Concepts. Consider the MIMO system of the form

$$
\left\{\begin{array}{l}
\dot{x}=f(u, x)+\bar{\varepsilon}(t), \\
y=\bar{C} x=x^{1},
\end{array}\right.
$$

with

$$
x=\left(\begin{array}{c}
x^{1} \\
x^{2} \\
\vdots \\
x^{q}
\end{array}\right) ; f(u, x)=\left(\begin{array}{c}
f^{1}\left(u, x^{1}, x^{2}\right) \\
f^{2}\left(u, x^{1}, x^{2}, x^{3}\right) \\
\vdots \\
f^{q-1}(u, x) \\
f^{q}(u, x)
\end{array}\right)
$$

$\bar{\varepsilon}(t)=\left(\begin{array}{c}0 \\ \vdots \\ 0 \\ \varepsilon(t)\end{array}\right), \varepsilon=\left(\begin{array}{c}\varepsilon_{1} \\ \vdots \\ \varepsilon_{n_{q}}\end{array}\right)$; and $\bar{C}=\left[I_{n_{1}}, 0_{n_{1} \times n_{2}}, 0_{n_{1} \times n_{3}}, \ldots\right.$,

$\left.0_{n_{1} \times n q}\right]$, where the state $x \in \mathfrak{R}^{n}$ and $x^{k} \in \mathfrak{R}^{n_{k}}, k=1, \ldots, q$ and $p=n_{1} \geq n_{2} \geq \cdots \geq n_{q}, \sum_{k=1}^{q} n_{k}=n$; the input $u(t) \in U$ is a compact subset of $\mathfrak{R}^{s} ; f(u, x) \in \mathfrak{R}^{n}$ with $f^{k}(u, x) \in \mathfrak{R}^{n_{k}}$; and $\bar{\varepsilon}(t) \in \mathfrak{R}^{n}$, where $\varepsilon(t) \in \mathfrak{R}^{n_{q}}$ with each $\varepsilon_{i}, i=1, \ldots, n_{q}$, being an unknown bounded real-valued function which may depend on $x, u$, uncertain parameters.

3.2.2. Observer Design. Consider the following change of coordinates:

$$
\begin{gathered}
\Phi: \Re^{n} \longrightarrow \mathfrak{R}^{n_{1 q}}, \\
\Phi=\left(\begin{array}{c}
x^{1} \\
x^{2} \\
\vdots \\
x^{q}
\end{array}\right) \longrightarrow z=\left(\begin{array}{c}
z^{1} \\
z^{2} \\
\vdots \\
z^{q}
\end{array}\right) ; \\
x^{1} \\
f^{1}\left(u, x^{1}, x^{2}\right) \\
\frac{\partial f^{1}}{\partial x^{2}}\left(u, x^{1}, x^{2}\right) f^{2}\left(u, x^{1}, x^{2}, x^{3}\right) \\
\vdots \\
\left(\begin{array}{l}
q-2 \\
k=1
\end{array}\right),
\end{gathered}
$$

where $z^{k} \in \mathfrak{R}^{n_{1}}, k=1, \ldots, q$.

Let us introduce the following notations:

$$
\begin{aligned}
\Lambda(u, x)= & \operatorname{diag}\left(I_{n_{1}}, \frac{\partial f^{1}}{\partial x^{2}}(u, x), \frac{\partial f^{1}}{\partial x^{2}}(u, x) \frac{\partial f^{2}}{\partial x^{3}}(u, x), \ldots,\right. \\
& \left.\prod_{k=1}^{q-1} \frac{\partial f^{k}}{\partial x^{k+1}}(u, x)\right) .
\end{aligned}
$$

According to Farza et al. [16], the transformation $\Phi(\cdot)$ puts system (6) under the following form: 


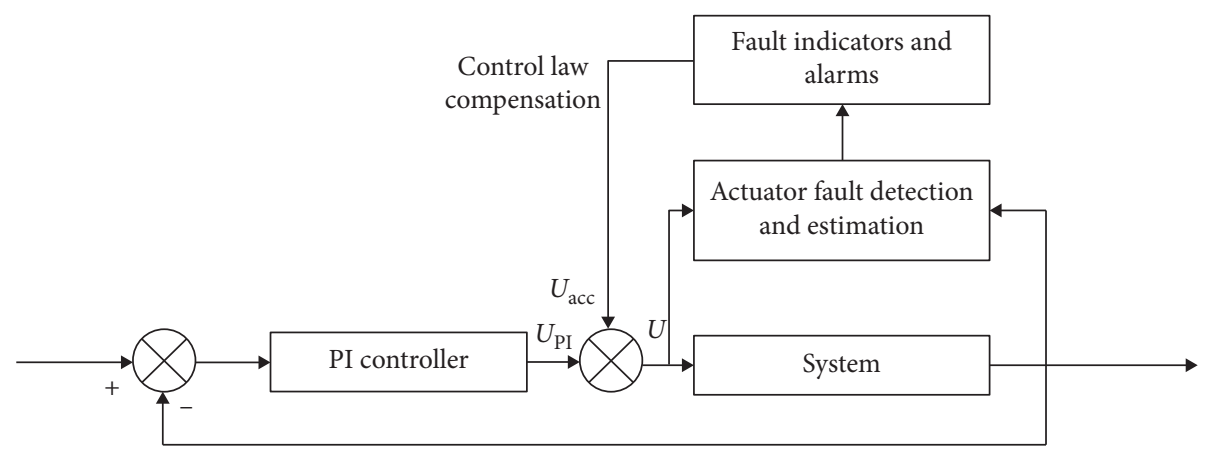

FIgUre 2: Synoptic scheme of FDE applied to 3TS.

$$
\left\{\begin{array}{l}
\dot{z}=A z+\varphi(u, z)+\frac{\partial \Phi}{\partial x}(u, x) \bar{\varepsilon}(t) \\
y=C z=z^{1}
\end{array}\right.
$$

where $\varphi(u, z)$ has a triangular structure:

$$
\varphi(u, z)=\left(\begin{array}{c}
\varphi^{1}\left(u, z^{1}\right) \\
\varphi^{2}\left(u, z^{1}, z^{2}\right) \\
\vdots \\
\varphi^{k}\left(u, z^{1}, \ldots, z^{k}\right) \\
\vdots \\
\varphi^{q}(u, z)
\end{array}\right),
$$

with $\varphi^{k}(u, z) \in \Re^{n_{1}}, k=1, \ldots, q$, and $C=\left[I_{n_{1}}, 0_{n_{1}}, \ldots, 0_{n_{1}}\right]$ is the $n_{1} \times n_{1 q}$ matrix with $0_{n_{1}}$ denoting the $n_{1} \times n_{1}$ null matrix.

3.2.3. Observer Synthesis. As in many works related to highgain observer synthesis, the observer design requires the following assumption [15].

A1: each function $f^{k}(u, x), k=1, \ldots, q-1$ satisfies the following rank condition.

$\operatorname{Rank}\left(\left(\partial f^{k} / \partial x^{k+1}\right)(u, x)\right)=n_{k+1}, \forall x \in \mathbb{R}^{n} ; \forall u \in U ;$ moreover, $\exists \alpha, \beta>0$ such that, for all $k \in\{1, \ldots$, $q-1\}, \forall x \in \mathbb{R}^{n} ; \forall u \in U$,

$$
\alpha^{2} I_{n k+1} \leq\left(\frac{\partial f^{k}}{\partial x^{k+1}}(u, x)\right)^{T} \frac{\partial f^{k}}{\partial x^{k+1}}(u, x) \leq \beta^{2} I_{n k+1},
$$

where $I_{n k+1}$ is the $n_{k+1} \times n_{k+1}$ identity matrix.

A2: the functions $\varphi^{i}(\cdot)$ for $i \in[1, q]$ are globally Lipschitz with respect to $x$ uniformly in $u$, i.e., $\exists L>0$, such that the following inequality holds for $i \in[1, q]$ and $x$ and $\bar{x} \in \mathbb{R}^{n}$ :

$$
\left\|\varphi^{i}(u, x)-\varphi^{i}(u, \bar{x})\right\| \leq L\|x-\bar{x}\| .
$$

The candidate observer for system (7) can be specified as

$$
\begin{aligned}
\dot{\hat{z}}= & A z+\varphi(u, \widehat{z})-\theta \Delta_{\theta}^{-1} S^{-1} k(\widehat{z})-\frac{\partial \varphi}{\partial x}\left(u, \Phi^{c}(\widehat{z})\right) . \\
& \cdot\left(\Lambda^{+}\left(u, \Phi^{c}(\widehat{z})\right)-\left(\frac{\partial \Phi}{\partial x}\left(u, \Phi^{c}(\widehat{z})\right)\right)^{+}\right) \theta \Delta_{\theta}^{-1} S^{-1} k\left(\widetilde{z}^{1}\right),
\end{aligned}
$$

where $\widehat{z}=\left(\begin{array}{c}\widehat{z}^{1} \\ \widehat{z}^{2} \\ \vdots \\ \widehat{z}^{q}\end{array}\right) \in \mathfrak{R}^{n_{1} q}, \widehat{z}^{k} \in \mathfrak{R}^{n_{1}}, k=1, \ldots, q ; S$ and $\Delta_{\theta}$ are given by $\Delta_{\theta}=\operatorname{diag}\left[I_{n_{1}},(1 / \theta) I_{n_{1}}, \ldots,\left(1 / \theta^{q-1}\right) I_{n_{1}}\right]$, where $\theta>0$ is a real number.

$S$ is the unique solution of the algebraic Lyapunov equation $S+A^{T} S+S A-C^{T} C=0$, where $A$ and $C$ are, respectively, given by

$$
\begin{aligned}
A & =\left[\begin{array}{ccccc}
0 & I_{n_{1}} & 0 & . & 0 \\
: & \cdot & I_{n_{1}} & \ddots & \cdot \\
0 & \ddots & \cdot & \cdot & 0 \\
0 & \ddots & \ddots & \cdot & I_{n_{1}} \\
0 & \cdot & . & 0 & 0
\end{array}\right] ; \\
C & =\left[I_{n_{1}}, 0_{n_{1}}, \ldots, 0_{n_{1}}\right], \\
\tilde{z} & =\widehat{z}-z .
\end{aligned}
$$

The proof of estimation error convergence is detailed in [16].

3.2.4. Observer Equations in the Original Coordinates. The observer (10) can also be given in the original coordinates $x$ as follows:

$$
\dot{\hat{x}}=f(u, \widehat{x})-\theta \Lambda^{+}(u, \widehat{x}) \Delta_{\theta}^{-1} S^{-1} k\left(\tilde{x}^{1}\right)
$$

where $S, C, \Delta_{\theta}$, and $\Lambda^{+}(u, x)$ are given above and $\widehat{x}=\left(\begin{array}{c}\hat{x}^{1} \\ \widehat{x}^{2} \\ \vdots \\ \hat{x}^{q}\end{array}\right) \in \mathfrak{R}^{n}$ with $\hat{x}^{k} \in \mathfrak{R}^{n_{k}}, k=1, \ldots, q ; u$ is the input of the system, and $\tilde{x}=\hat{x}-x$. 


\section{FTC Strategy}

The main objective is to establish a closed-loop regulation to track two reference levels. For this reason, two PI controllers are designed. Each one controls one liquid level. In the safety mode, these controllers can successfully accomplish this task. However, in the case of an actuator fault, nominal performances are affected in the best case, and it can lead to instability in the worst case. To avoid such behaviour, we should use the FTC that allows the accommodation of the control law by the addition of the estimated actuator fault magnitude to the nominal control law. As it is shown in Figure 2, the FTC can be divided into two main tasks: the first one is the FDIE detailed in the third paragraph, and the second one concerns the control law reconfiguration. Once the FDIE is performed, the faulty actuator $A_{i}$ is identified and isolated, and the residual variable $R_{i}$, different to zero can be used as a fault indicator or alarm. To reduce the effect of the actuator fault and to maintain the closed-loop performance, the actuator fault estimated previously is added to the nominal control law.

4.1. Design of the FTC System for Actuator Faults. Consider a nonlinear system given by

where $x=\left(\begin{array}{c}x^{1} \\ x^{2} \\ \vdots \\ x^{q}\end{array}\right)$ is the state vector. form:

In case of actuator faults, system (17) has the following

$$
\left\{\begin{array}{l}
\dot{x}=f(x)+g \cdot u+l \cdot u_{f} \\
y=c \cdot x
\end{array}\right.
$$

In this work, compensation to the control law for the faulty system is added, and the new control law is given by

$$
u_{\mathrm{FTC}}=u+u_{\mathrm{acc}}
$$

where $u$ is the nominal control law and $u_{\text {acc }}$ is the accommodation for the control law; system (18) becomes

$$
\left\{\begin{array}{l}
\dot{x}=f(x)+g \cdot u+g \cdot u_{\mathrm{acc}}+l \cdot u_{f}, \\
y=c \cdot x
\end{array}\right.
$$

where additional action must be quickly computed such as the system is recovered as soon as possible; then, the following condition is requested:

$$
\begin{aligned}
g \cdot u_{\mathrm{acc}}+l \cdot u_{f} & =0 ; \\
u_{\mathrm{acc}} & =-g^{+} \cdot l \cdot u_{f},
\end{aligned}
$$

where $g^{+}$is the pseudo-inverse matrix of $g$ and $u_{f}$ is the fault actuator estimation.

4.2. Application to the Three-Tank System. The mathematic model of hydrographic system (1) can be written in the following form:

$$
\left\{\begin{array}{l}
\dot{h}=f(h)+g \cdot Q \\
y=C \cdot h
\end{array}\right.
$$

where $h=\left[\begin{array}{l}h_{1} \\ h_{2} \\ h_{3}\end{array}\right], Q=\left[\begin{array}{l}Q_{1} \\ Q_{2}\end{array}\right], g=\left[\begin{array}{cc}1 / S & 0 \\ 0 & 0 \\ 0 & 1 / S\end{array}\right]$, and $f(h)=$

$$
\left[\begin{array}{c}
-a_{1} \operatorname{sgn}\left(h_{1}-h_{2}\right) \sqrt{\left|h_{1}-h_{2}\right|} \\
a_{1} \operatorname{sgn}\left(h_{1}-h_{2}\right) \sqrt{\left|h_{1}-h_{2}\right|}-a_{2} \operatorname{sgn}\left(h_{2}-h_{3}\right) \sqrt{\left|h_{2}-h_{3}\right|} \\
a_{2} \operatorname{sgn}\left(h_{2}-h_{3}\right) \sqrt{\left|h_{2}-h_{3}\right|}-a_{3} \sqrt{h_{3}}
\end{array}\right] .
$$

When the system is affected by the actuator fault, the mathematical model is given by

$$
\left\{\begin{array}{l}
\dot{h}=f(h)+g \cdot Q+l \cdot Q_{f} \\
y=C \cdot h
\end{array}\right.
$$

where $l=\left[\begin{array}{cc}1 / S & 0 \\ 0 & 0 \\ 0 & 1 / S\end{array}\right]$ and $Q_{f}=\left[\begin{array}{ll}Q_{f 1} & Q_{f 2}\end{array}\right]^{T}$.

To estimate the actuator fault denoted by $Q_{f 1}$ and $Q_{f 2}$, we use the high-gain observer as follows:

$$
\dot{\hat{x}}=f(u, \widehat{x})-K_{\theta} \Lambda^{+}(u, \widehat{x})\left(\tilde{x}^{1}\right),
$$

where

$$
\begin{aligned}
& x=\left[\begin{array}{ll}
x^{1} & x^{2}
\end{array}\right]^{T} ; \\
& x^{1}=\left[\begin{array}{lll}
h_{1} & h_{2} & h_{3}
\end{array}\right]^{T} \text {; } \\
& x^{2}=\left[\begin{array}{ll}
Q_{f 1} & Q_{f 2}
\end{array}\right]^{T} \text {; } \\
& f(u, \widehat{x})=f(\widehat{h})+g \cdot Q+l \cdot \widehat{Q}_{f}, \\
& K_{\theta}=\left[\begin{array}{ccccc}
2 \theta & 0 & 0 & 0 & 0 \\
0 & 2 \theta & 0 & 0 & 0 \\
0 & 0 & 2 \theta & 0 & 0 \\
0 & 0 & 0 & \theta^{2} & 0 \\
0 & 0 & 0 & 0 & \theta^{2}
\end{array}\right], \\
& \Lambda^{+}(u, \widehat{x})=\left[\begin{array}{lll}
1 & 0 & 0 \\
0 & 1 & 0 \\
0 & 0 & 1 \\
S & 0 & 0 \\
0 & 0 & S
\end{array}\right] \text {, } \\
& \tilde{x}^{1}=\hat{x}^{1}-x^{1} \text {. }
\end{aligned}
$$

To compensate for the fault actuator as mentioned above, a new control law is applied: $Q_{\mathrm{FTC}}=Q+Q_{\text {acc }}$.

The compensated mathematical model is given by

$$
\left\{\begin{array}{l}
\dot{h}=f(h)+g \cdot Q+g \cdot Q_{\mathrm{acc}}+l \cdot Q_{f}, \\
y=C \cdot h .
\end{array}\right.
$$

To compensate for the fault actuator, accommodation law must verify $Q_{\mathrm{acc}}=-g^{+} \cdot l \cdot Q_{f}$.

Remark 1. A main feature of the framework is given in [16] that the state observation scheme is based on a nonlinear 
class of systems, under which it belongs to the model of the three-tank process. We exploit the solid theoretical basis of the HGO approach to estimate not only the state vector but also the actuator fault. Indeed, the standard state vector (composed of the three liquid levels) is extended to both actuator faults (flow rates) so that the order of the system becomes five instead of three. Thereafter, once the new state vector is reconstructed by the HGO, it is easy to derive the estimation of the flow rates from its two last components.

\section{FTC Application}

To prove the validity of the FTC strategy proposed in Sections 3 and 4, we apply it to the three-tank system described in the Section 2. The aim is to accomplish a closedloop regulation of two levels $h_{1}$ and $h_{3}$ in the presence of actuator faults described by the following scenario. An interface card is used to establish the connection, via the USB port, between the control desk using the Matlab/Simulink environment and the three piezoresistive differential pressure sensors as the input and the motor pump drive board as outputs. Each sensor and its conditioner card deliver a voltage varying between 0 and $5 \mathrm{~V}$ corresponding, respectively, to a liquid height of 0 to $100 \mathrm{~cm}$. Using the PWM interface in Simulink library, the interface card associated with the dual-full bridge LM298 applies to the 2 pumps a variable voltage from 0 to $+12 \mathrm{~V}$, corresponding to a flow rate ranging from 0 to $Q_{\max }$.

The bench test used in this implantation, shown in Figure 3, is in the laboratory "Study of Industrial Systems and Renewable Energies," "ESIER," at the National Engineers School of Monastir, Tunisia.

\subsection{Fault Scenario}

(i) At $t_{1}=300 \mathrm{~s}$, a constant offset of $-4.2 \mathrm{~V}$ is added to the applied control law $u_{1}\left(\alpha 1=1, u_{10}=-4.2 \mathrm{~V}\right)$

(ii) At $t_{2}=350 \mathrm{~s}$, a constant offset of $-3.5 \mathrm{~V}$ is added to the applied control law $u_{2}\left(\alpha_{2}=1, u_{20}=-3.5 \mathrm{~V}\right)$

5.2. Experimental Results. To highlight the proposed AFTC, we compare the tracking performance of the two levels $h_{1}$ and $h_{3}$ in case A and case B.

In case $A$, we use a feedback regulation for the two liquid levels based on a PI controller, which means that the process acts without AFTC. In case B, in addition to the PI controller, we use the AFTC module whose role is to add a compensation control law.

As it is shown in Figures 4 and 5, each controlled level decreases, after fault occurrence, to reach a minimum value and then it reaches the desired value after a time delay relative to each case.

The minimum value reached in case A is $39.39 \mathrm{~cm}$ for $h_{1}$ and $26.41 \mathrm{~cm}$ for $h_{3}$, while in case B, it reached $39.79 \mathrm{~cm}$ and $26.69 \mathrm{~cm}$, respectively, for $h_{1}$ and $h_{3}$ as it is mentioned in Table 1.

In case $\mathrm{A}$, the PI controller will react to compensate for the fault effect after a time delay of $17.7 \mathrm{~s}$ for $h_{1}$ and $20.1 \mathrm{~s}$ for

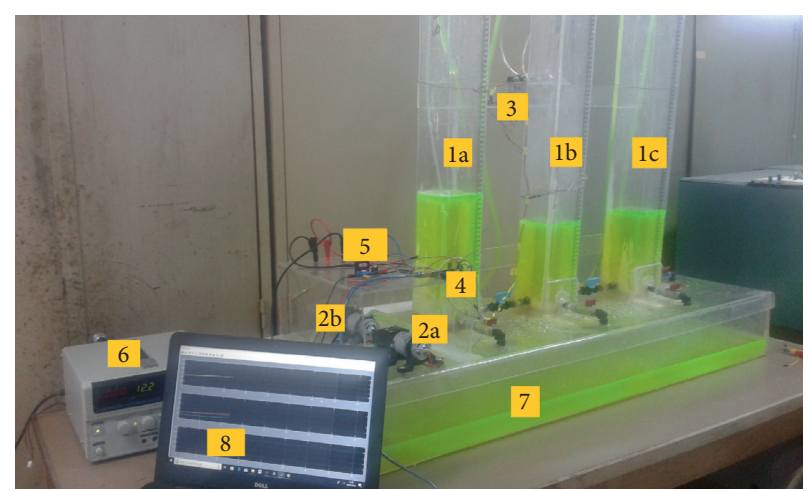

FIgURE 3: Experimental plant: (1a, 1b, and 1c) 3 cubic tanks; (2a and 2b) 2 DC motor pumps; (3) liquid level sensors; (4) Fio std STM32F10 board; (5) motor driver board; (6) power supply; (7) basin; (8) control desk.

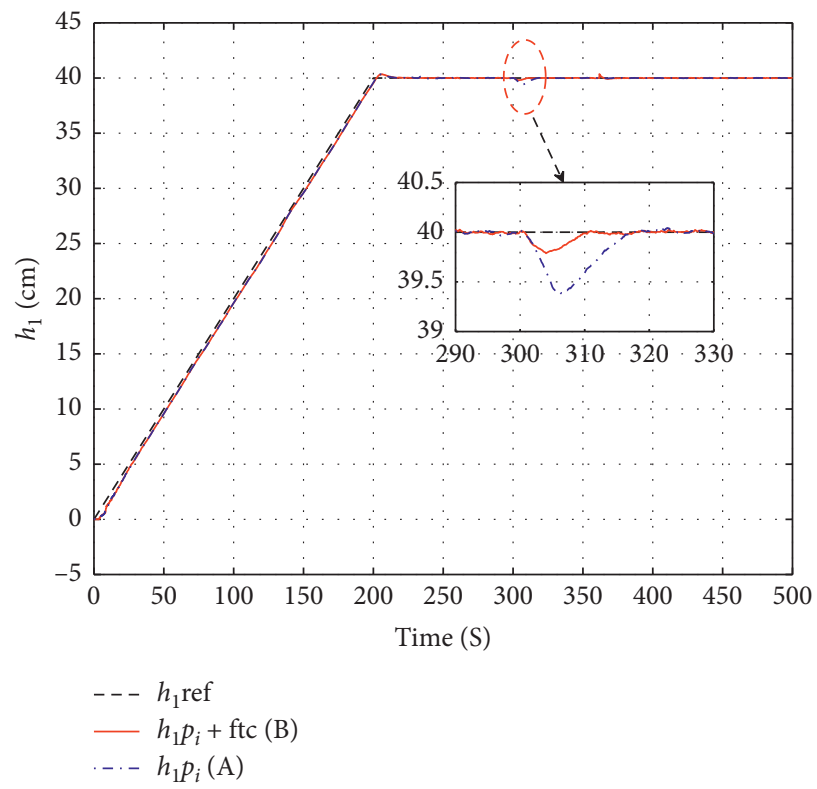

FIgURE 4: Tracking performance of level 1 in cases A and B.

$h_{3}$; However, in case $\mathrm{B}$, the controlled level reached the desired value after $10.6 \mathrm{~s}$ and $10.8 \mathrm{~s}$, respectively, for $h_{1}$ and $h_{3}$.

To evaluate the control performance, we use the mean square error (MSE) criterion defined by

$$
\mathrm{MSE}=\frac{1}{n} \sum_{k=1}^{n}\left(y_{\mathrm{ref}}(k)-y(k)\right)^{2},
$$

where $n$ is the number of measurements, $y_{\text {ref }}(k)$ is the desired output, and $y(k)$ is the system output. The computation of the MSE for outputs $y_{1}$ and $y_{3}$ in cases A and B is given by Table 1 .

As it is shown in Table 1, the MSE of the proposed AFTC approach is smaller than case A when the PI controller acts only to compensate for the actuator fault.

It can be easily seen in Table 1 that the fault detection time $t_{d}$ in case $\mathrm{B}$ is smaller than case $\mathrm{A}$ for both liquid levels. 


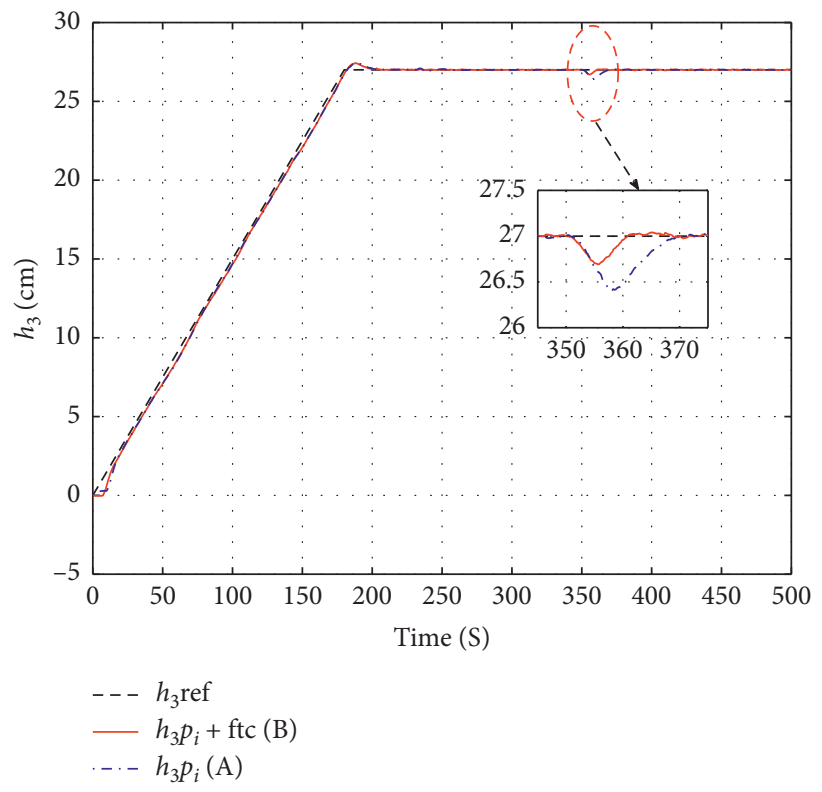

Figure 5: Tracking performance of level 3 in cases A and B.

TABLE 1: Tracking performance comparison.

\begin{tabular}{ccccccccccccc}
\hline \multicolumn{1}{c}{ Case A (without AFTC) } & \multicolumn{4}{c}{ Case B (with AFTC) } \\
\hline \multirow{2}{*}{$h_{1}$} & $h_{1 n}$ & $h_{1 \mathrm{~min}}$ & $t_{f}$ & $t_{d}$ & $t_{\text {comp }}$ & MSE & $h_{1 n}$ & $h_{1 \mathrm{~min}}$ & $t_{f}$ & $t_{d}$ & $t_{\text {comp }}$ & MSE \\
& $40 \mathrm{~cm}$ & $39.39 \mathrm{~cm}$ & $300 \mathrm{~s}$ & 306.5 & $317.7 \mathrm{~s}$ & 0.0873 & $40 \mathrm{~cm}$ & $39.79 \mathrm{~cm}$ & $300 \mathrm{~s}$ & 303.5 & $310.6 \mathrm{~s}$ & 0.0824 \\
\hline \multirow{2}{*}{$h_{3}$} & $h_{3 n}$ & $h_{3 \mathrm{~min}}$ & $t_{f}$ & $t_{d}$ & $t_{\text {comp }}$ & MSE & $h_{3 n}$ & $h_{3 \mathrm{~min}}$ & $t_{f}$ & $t_{d}$ & $t_{\text {comp }}$ & MSE \\
& $27 \mathrm{~cm}$ & $26.41 \mathrm{~cm}$ & $350 \mathrm{~s}$ & 358 & $370.1 \mathrm{~s}$ & 0.0614 & $27 \mathrm{~cm}$ & $26.69 \mathrm{~cm}$ & $350 \mathrm{~s}$ & 355.7 & $360.8 \mathrm{~s}$ & 0.0549 \\
\hline
\end{tabular}

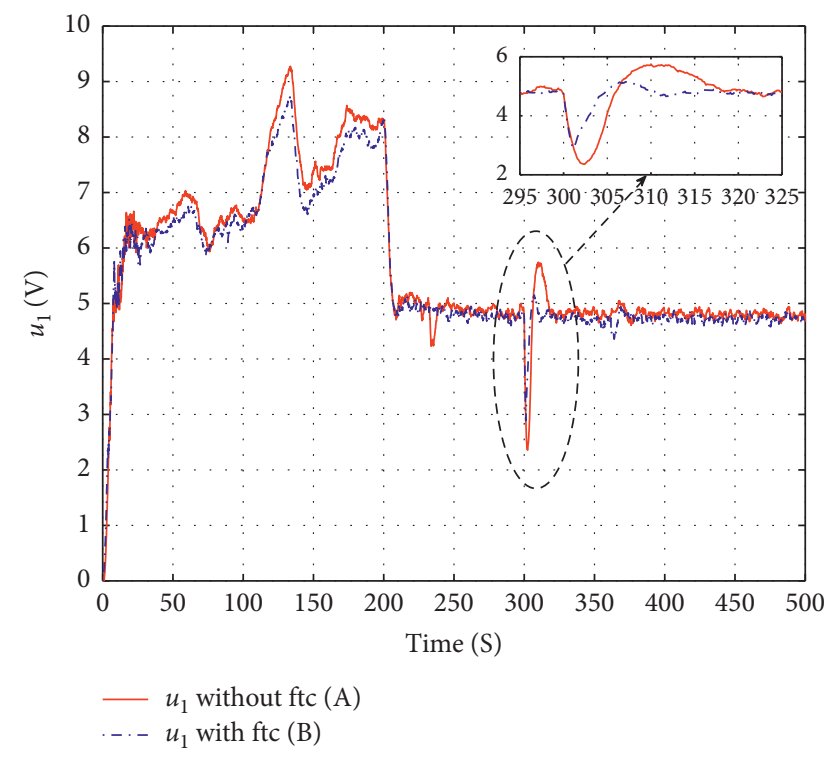

Figure 6: The control law applied to pump 1 in cases A and B.

Compared to the work given in [14], the proposed AFTC has significant merit when comparing the fault detection time $t_{d}$ which decreases from $16 \mathrm{~s}$ to $3.3 \mathrm{~s}$.

In Figures 6 and 7, the control laws applied to motor pumps increase slowly after fault occurrence in case $\mathrm{A}$, but in

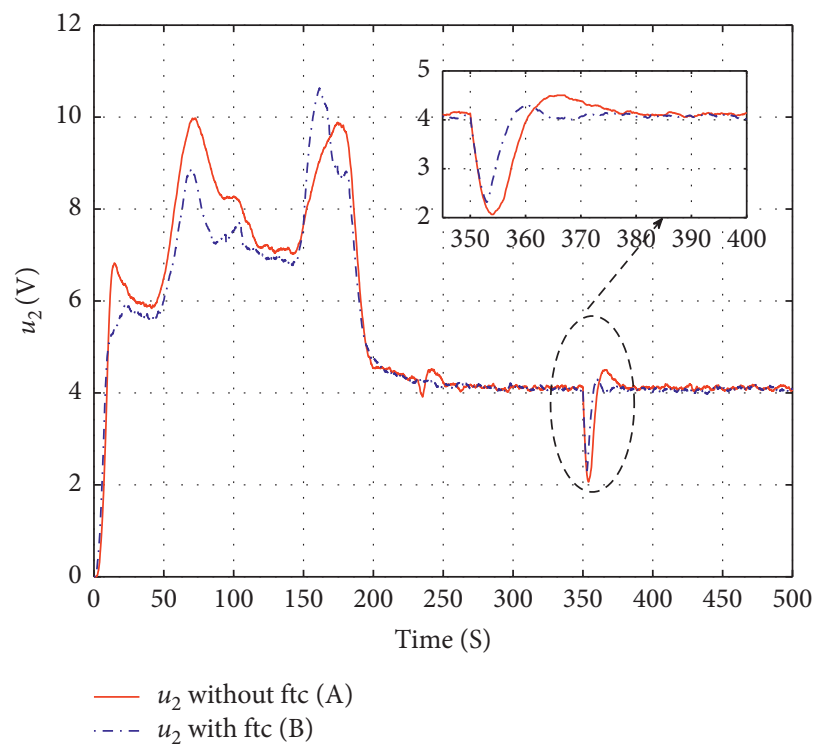

Figure 7: The control law applied to pump 2 in cases A and B.

case B, the control law increases quicker and enables the fast fault compensation with a smaller overshoot.

In Figures 8 and 9, the control law generated by the PI controller in case A increases suddenly after fault occurrence in order to compensate the actuator degradation. But, in case 


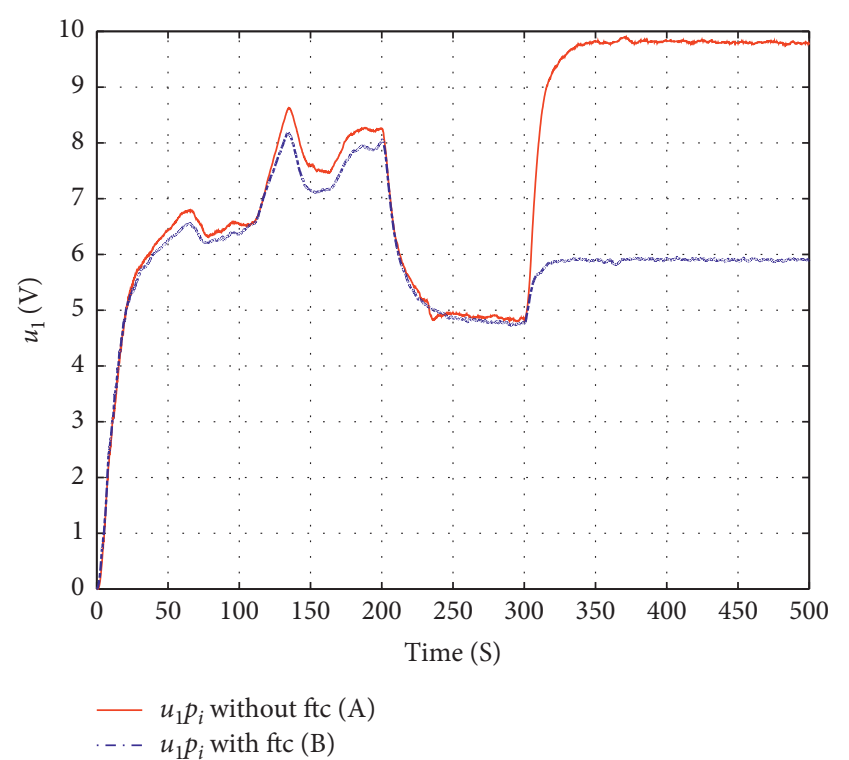

Figure 8: The PI control law applied to pump 1 in cases A and B.

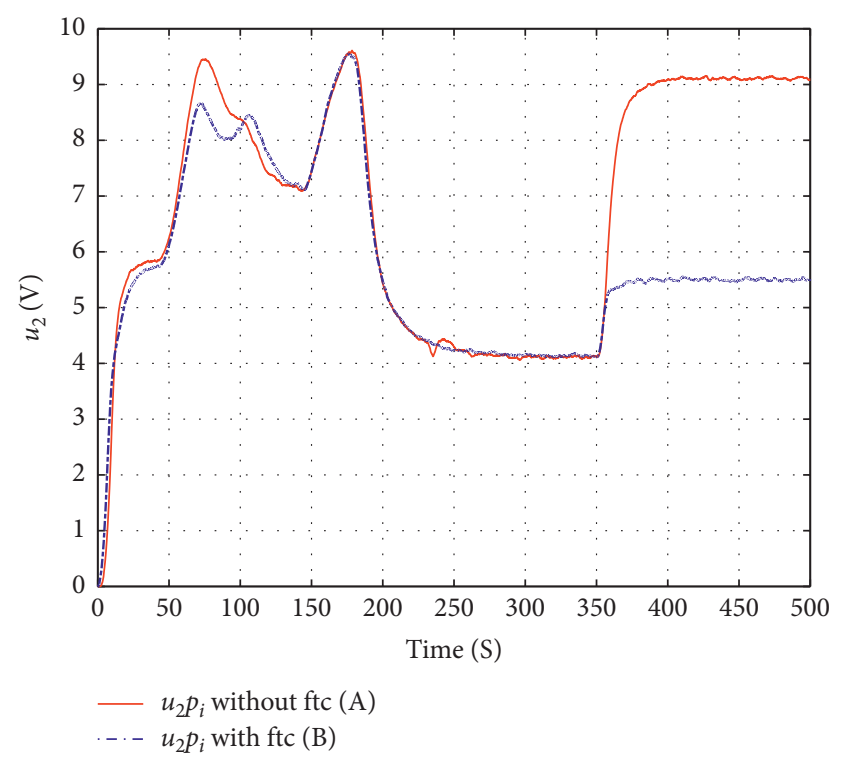

Figure 9: The PI control law applied to pump 2 in cases A and B.

$\mathrm{B}$, the generated PI controller signal is little greater than the nominal control law (fault-free case) because of the estimated actuator fault magnitude injection.

In this experimental task, the operating point is well chosen so that the PI controller can compensate for the fault effect. For this same operating point and fault magnitude, the proposed approach AFTC in case B can compensate easily for the actuator fault. This is another argument that highlights the proposed AFTC.

\section{Conclusion}

In this paper, a high-gain observer is used to estimate the actuator fault magnitude applied to a three-tank system. In the faulty case, the estimated fault is added to the control law in order to compensate for the fault effect as quickly as possible. Compared to the faulty outputs with a classic PI controller, experimental results show that the compensated output behaviour is closer to the fault-free outputs. This work can be extended using an adaptive observer whose role is to estimate both the unknown output and the unavailable state variable for measurement. Fault sensor reconstruction is also one of our future interests.

\section{Data Availability}

The data used to support the findings of this study are available from the corresponding author upon request.

\section{Conflicts of Interest}

The authors declare that they have no conflicts of interest.

\section{References}

[1] A. Zolghadri, J. Cieslak, D. Efimov et al., "Signal and modelbased fault detection for aircraft systems," IFAC-Papers Online, vol. 48, no. 21, pp. 1096-1101, 2015.

[2] D. Zhihong, S. Xiaocheng, X. Guoqing, and F. Mingyu, "Fault tolerant control for steam generators in nuclear power plant," Nuclear Power Engineering, vol. 31, no. 1, pp. 107-111, 2010.

[3] J. M. Gregor and A. Cinar, "Monitoring, fault diagnosis, faulttolerant control and optimization: data driven methods," Computers and Chemical Engineering, vol. 47, no. 20, pp. 111-120, 2012.

[4] A. Pantea, A. Yazidi, F. Betin et al., "Fault tolerant control of a low speed six-phase induction generator for wind turbines"” IEEE Transactions on Industry Applications, vol. 55, no. 1, pp. 426-436, 2019.

[5] D. Koenig, S. Nowakowski, and T. Cecchin, "An original approach for actuator and component fault detection and isolation," in Proceedings of the 3rd IFAC Symposium on Fault Detection Supervision and Safety for Technical Processes, pp. 95-105, Hull, UK, 1997.

[6] C. Join, J.-C. Ponsart, D. Sauter, and D. Theilliol, "Nonlinear filter design for fault diagnosis: application to the three-tank system," IEE Proceedings-Control Theory and Applications, vol. 152, no. 1, pp. 55-64, 2005.

[7] A. Akhenak, M. Chadli, D. Maquin, and J. Ragot, "State estimation via multiple observer the three-tank system," in Proceedings of the 5th IFAC Symposium on Fault Detection, Supervision and Safety for Technical Processes, pp. 1227-1232, Washington DC, USA, 2003.

[8] M. Rodrigues, D. Theilliol, M. Adam-Medina, and D. Sauter, "A fault detection and isolation scheme for industrial systems based on multiple operating models," Control Engineering Practice, vol. 16, no. 2, pp. 225-239, 2008.

[9] M. Amor, T. Ladhari, S. Hadj Said, and F. M'Sahli, "Hardware implementation of actuator fault estimation applied to a hydrographic plant," in Proceedings of the 19th International Conference on Sciences and Techniques of Automatic Control \& Computer Engineering (STA), pp. 202-207, Sousse, Tunisia, March 2019.

[10] L. F. Mendonc, J. M. C. Sousa, and J. M. G. Sa da Costa, "Fault accommodation of an experimental three tank system using fuzzy predictive control," in Proceedings of the 2008 IEEE 
International Conference on Fuzzy Systems, Hong Kong, China, June 2008.

[11] M. T. César, L. Loïc, C. Franck, A. G. Efraín, and D. R. David, "Flatness-based fault tolerant control," DYNA, vol. 81, no. 188, pp. 131-138, 2014.

[12] R. Seydou, T. Raissi, A. Zolghadri, and D. Efimov, "Actuator fault diagnosis for flat systems: a constraint satisfaction approach," International Journal of Applied Mathematics and Computer Science, vol. 23, no. 1, pp. 171-181, 2013.

[13] H. Noura, D. Theilliol, and D. Sauter, "Actuator fault-tolerant control design: demonstration on a three-tank-system," International Journal of Systems Science, vol. 31, no. 9, pp. 1143-1155, 2000.

[14] D. Theilliol, H. Noura, and J.-C. Ponsart, "Fault diagnosis and accommodation of a three-tank system based on analytical redundancy," ISA Transactions, vol. 41, no. 3, pp. 365-382, 2002.

[15] M. Amor, T. Ladhari, S. Hadj Said, and F. M'Sahli, "On-line continuous-discrete time observer for three-tank system," in Proceedings of the 15th International Multi-Conference on Systems, Signals \& Devices (SSD), pp. 566-571, Hammamet, Tunisia, March 2018.

[16] M. Farza, M. M'Saad, and M. Sekher, "A set of observers for a class of nonlinear systems," in Proceedings of the 16th IFAC World Congress, Praha, Czech Republic, July 2005. 